\title{
Predicting dementia using socio- demographic characteristics and the Free and Cued Selective Reminding Test in the general population
}

Thibault Mura ${ }^{1,2,3^{*}}$ (D), Marieta Baramova ${ }^{1}$, Audrey Gabelle ${ }^{1,2,4}$, Sylvaine Artero', Jean-François Dartigues ${ }^{5,6,7}$, Hélène Amieva ${ }^{6,7}$ and Claudine Berr ${ }^{1,2}$

\begin{abstract}
Background: Our study aimed to determine whether the consideration of socio-demographic features improves the prediction of Alzheimer's dementia (AD) at 5 years when using the Free and Cued Selective Reminding Test (FCSRT) in the general older population.

Methods: Our analyses focused on 2558 subjects from the prospective Three-City Study, a cohort of communitydwelling individuals aged 65 years and over, with FCSRT scores. Four "residual scores" and "risk scores" were built that included the FCSRT scores and socio-demographic variables. The predictive performance of crude, residual and risk scores was analyzed by comparing the areas under the ROC curve (AUC).

Results: In total, 1750 subjects were seen 5 years after completing the FCSRT. AD was diagnosed in 116 of them. Compared with the crude free-recall score, the predictive performances of the residual score and of the risk score were not significantly improved (AUC: 0.83 vs 0.82 and 0.88 vs 0.89 respectively).
\end{abstract}

Conclusion: Using socio-demographic features in addition to the FCSRT does not improve its predictive performance for dementia or AD.

Keyword: Dementia, Prediction, Alzheimer disease, prodromal Alzheimer disease, preclinical Alzheimer disease, Early clinical trial

\section{Background}

The early identification of preclinical forms of Alzheimer's disease $(\mathrm{AD})$ has been the focus of much research over the last two decades. Indeed, because the $\mathrm{AD}$ pathophysiological process begins several years or decades before the clinical diagnosis [1-3], it seems more promising, for treating the disease, to act upstream of the clinical stage, before the installation of irreversible damage [4]. The development of such therapies requires the early identification of patients with $\mathrm{AD}$ at the predementia stage. In accordance with these conceptual

\footnotetext{
* Correspondence: t-mura@chu-montpellier.fr

${ }^{1}$ Neuropsychiatry: Epidemiological and Clinical Research, INSERM U1061, 34093 Montpellier, Cedex 5, France

2University of Montpellier, Montpellier, France

Full list of author information is available at the end of the article
}

advances, AD diagnosis has been redefined recently in the context of research. It now requires, for its typical form, the combination of amnesic syndrome of hippocampal type and a pathophysiological $\mathrm{AD}$ biomarker, such as cerebrospinal fluid proteins (decreased A $\beta 42$ and increased T-tau and P-tau) or amyloid plaques detected by PET imaging with a specific tracer [5]. However, the measurement of such biomarkers cannot be generalized to the entire population because of the potential side effects of the used methods (exposure to radiation for brain imaging; pain and risks related to invasive procedures for cerebrospinal fluid collection) and because of their ever-increasing cost [6]. A possible alternative could be a large screening using a noninvasive and cheap tool [7], such as a neuropsychological test. The Free and Cued Selective Reminding Test 
(FCSRT) [8], which has been recommended for assessing amnesic syndrome of hippocampal type $[5,9]$, could be potentially useful in this context. Indeed, in a first study conducted in general population [10], the FCSRT exhibited good sensitivity and specificity for AD prediction at 5 years (92\% and 64\% respectively for the FCSRT free recall), but showed a poor positive predictive value of about $8 \%$.

To our knowledge, no study has tried to improve the predictive performances of a neuropsychological test by combining the test results with some readily available information, such as socio-demographic data. Sociodemographic features (sex, age and education) strongly influence cognitive scores [11-16] and the dementia risk [17]. Combining these characteristics with the results of an episodic memory test could therefore improve dementia prediction. Two types of algorithm can be used for this prediction. The predictive information contained in socio-demographic variables can be added to the neuropsychological test score to generate a predictive "risk score", built according to the recommendations of the international literature [18]. Alternatively, an algorithm can be developed to allow the interpretation of the neuropsychological test scores as a function of the socio-demographic characteristics. A "residual score" can thus be calculated that corresponds to the difference between the observed and the expected scores for a subject of a given sex, age and education level. Such a "residual score" was recently proposed by Reed et al. [19] for quantifying the cognitive reserve [20] from the scores of an episodic memory test after removing the variability due to socio-demographic factors and level of brain pathology.

The objective of this study was therefore to determine whether the addition of socio-demographic factors to the FCSRT score to generate a risk score or the use of a residual score could improve the prediction of dementia and/or AD at 3 and 5 years compared with the FCSRT crude scores alone, in a population-based cohort of older subjects.

\section{Methods}

\section{Population and study design}

Data were extracted from the multi-site prospective Three-City Study (3C) cohort study on 9294 community-dwelling persons aged 65 years and over recruited from the electoral rolls of three French cities between 1999 and 2001 [21]. The 3C protocol was approved by the Ethical Committee of the University Hospital of Bicêtre (France) and written informed consent was obtained from each participant. Sociodemographic characteristics, health status and lifestyle information were collected using standardized questionnaires during face-to-face interviews. Subjects were seen again at 2 years (S1), 4 years (S2), 5 years (S3), 7 years (S4), 10 years (S5) and 12 years (S6) after inclusion. Because the FCSRT was administered only to participants from the Montpellier and Bordeaux $3 \mathrm{C}$ centers at the S4 visit, the current study used data for 2558 subjects from these two cities who were nondemented and completed the cognitive tests at S4 (baseline of our analyses). Among these subjects, 459 were excluded (170 dead and 289 lost to follow-up between S4 and S5) for the prediction of dementia at 3 years after FCSRT completion, and 808 (351 dead and 457 lost to follow-up between S4 and S6) for the prediction of dementia at 5 years after the FCSRT (Fig. 1).

\section{FCSRT administration and scores}

The French version [22] of the FCSRT $[8,22]$ was administered at S4 for the first time in the Montpellier center and for the second time in the Bordeaux center. The test assesses verbal episodic memory. The neuropsychological test begins with an encoding phase during which the patient has to learn 16 words, four at a time (presented on a card). Each word belongs to a different semantic category. The subject is asked to say the name of the item corresponding to a specific semantic category (e.g., "what is the name of this fruit?"). After all four items are identified correctly, the card is removed and their immediate cued recall is tested by presenting the cues again in order to control for encoding. Once the immediate recall of a group of four items is completed, the next set of four items is presented. After the encoding phase is completed, the participant is asked to recall as many words as possible in $2 \mathrm{mi}$ nutes (free recall). The neuropsychologist then provides a cue (word category) for each nonretrieved word to help the patient recalling the remaining words. Free and cued recalls are repeated three times. The delayed recall phase is performed 20 minutes later, also with a free and a cued recall.

For our analyses, we used the "free recall score" (sum of the number of words retrieved at the three free recall trials), the "total recall score" (sum of the three free + cued recall trials) (both scores range from 0 to 48), the "delayed free recall score" (number of words retrieved at the delayed free recall trial) and the "delayed total recall score" (free + cued delayed recall trial) (both scores range from 0 to 16 ).

\section{Diagnosis of dementia}

Dementia was diagnosed in two steps at each follow-up visit. Subjects at risk were identified by the $3 \mathrm{C}$ neuropsychologist on the basis of the results of the MiniMental State Examination (MMSE) [23] and the Isaacs set test (IST) [24]. The IST, consisting of generating words belonging to four semantic categories (cities, 


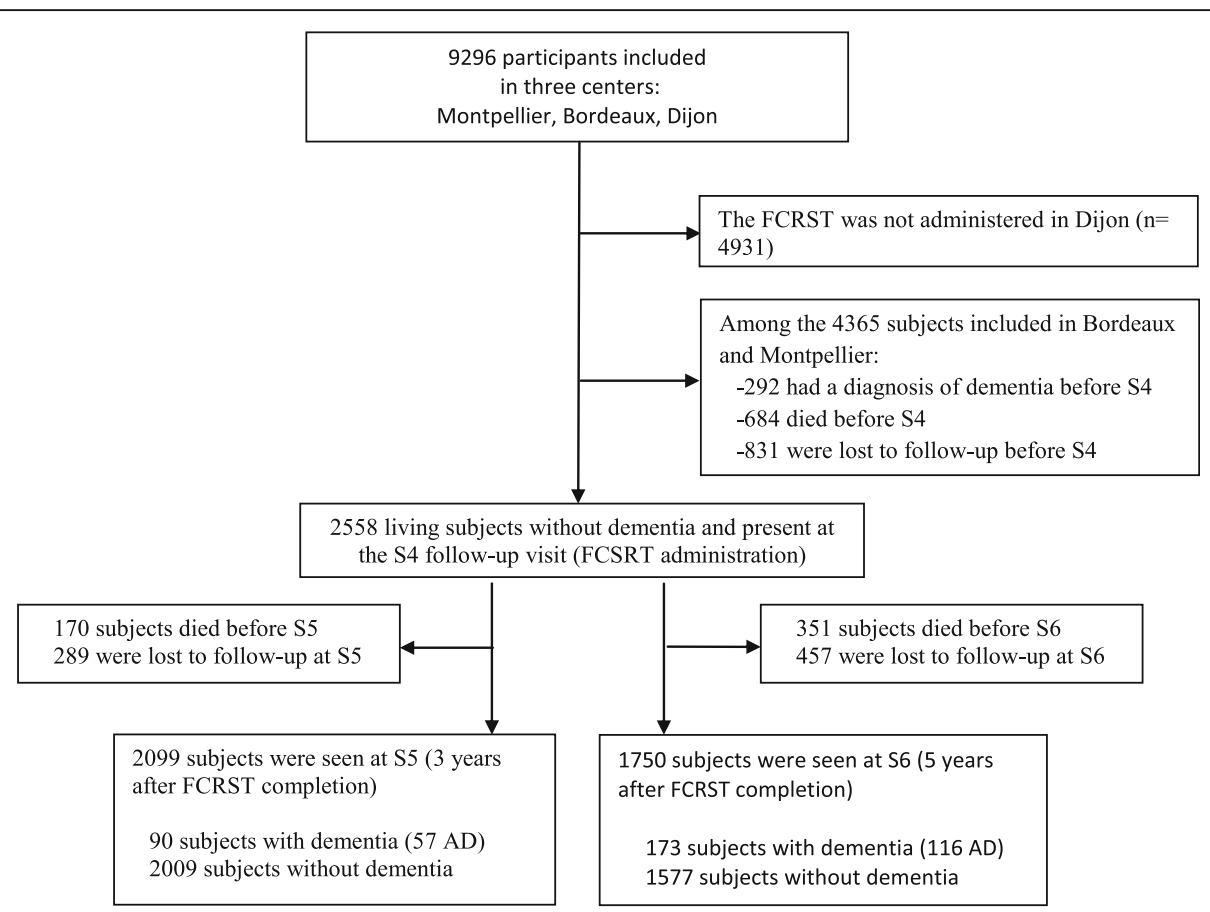

Fig. 1 Study flow chart. AD Alzheimer's disease, FCSRT Free and Cued Selective Reminding Test, S4 visit with FCRST administration, S5 3 years after FCRST administration, $S 65$ years after FCRST administration

fruits, animals and colors) in 15 seconds, measured semantic verbal fluency ability. These participants were then examined by a $3 \mathrm{C}$ physician who confirmed or not the diagnosis and severity of the disease. The physician was blind to the FCSRT scores. Finally, all diagnoses were reviewed by a panel of five neurologists, independent of the $3 \mathrm{C}$ investigators. The panel examined all available information, including the FCSRT scores, and agreed on the presence or absence of dementia, according to the DSM-IV TR criteria [25], and on its etiology using the National Institute of Neurological and Communicative Disorders and Stroke-Alzheimer's Disease and Related Disorders Association criteria [26] for probable and possible $\mathrm{AD}$. When we further analysed the prediction of $A D$, subjects with a diagnosis of non- $A D$ dementia were not excluded but were included in a group which contained both patients without dementia and patients with non-AD dementia.

\section{Statistical analysis}

The participants' characteristics were described using frequency and proportion for categorical variables and mean and standard deviation (SD) for continuous variables. Comparisons between groups were performed using the Student's $t$ test for the FCSRT scores and the chi-square test for percentages of missing data (subjects who did not begin or complete the FCSRT at S4).
To construct the FCSRT "residual scores", the crude scores were modeled using linear regression models that included, as independent variables, age, age-squared, sex and education level (primary school: $0-5$ years of education; vocational school certificate; French junior-school diploma; French high-school diploma; graduate studies). The total proportion of variance of the crude scores explained by the linear model was expressed using the coefficient of determination, denoted $R^{2}$. The estimated beta coefficients were used to generate the estimated FCSRT scores for each individual. The "residual scores" were then defined and computed as the difference between the estimated and the crude FCSRT scores. Consequently, the variability of these "residual scores" corresponds to the FCSRT residual variance after removing the part of variance associated with the socio-demographics variables [19].

The dementia and AD "risk scores" were constructed using a logistic regression model. The probability of dementia or $\mathrm{AD}$ at 3 and 5 years (S5 and S6 visits) after FCSRT completion, and between these two visits, was modeled as a function of the FCSRT crude scores, age, sex, education level and interaction between the FCSRT crude scores and the three socio-demographic characteristics (age, sex and education level). Regression coefficients were used to compute a risk score for each subject. To avoid over-fitting, the risk scores were evaluated using cross-validation methods by splitting the sample into two, according to the inclusion center [27]. 
Thus, the risk score coefficients were estimated using only the Bordeaux data (training dataset) because of the larger number of patients with dementia in this center. The risk score predictive performances were evaluated using the Montpellier data (testing dataset). A sensibility analysis was conducted by inverting the cities for the training and testing dataset.

The performance of the "crude scores", "residual scores" and "risk scores" for the prediction of dementia and $\mathrm{AD}$ at 3 and 5 years, and between 3 and 5 years, was assessed using receiver operating curve (ROC) analysis [28]. The area under the curve (AUC) values were compared using the nonparametric method described by DeLong et al. [29].

Analyses were performed with a bilateral alpha level of 0.05 using SAS software, version 9.1 (SAS Institute, Cary, NC, USA).

\section{Results}

At the S4 follow-up visit (baseline of our analyses), the mean age of the 2558 participants was 80 years (SD: 4.7), $63.8 \%$ were women, $27.3 \%$ had $0-5$ years of education, $17.0 \%$ had a French junior-school diploma (9 years of education), $11.1 \%$ had a vocational school certificate (10-11 years of education), $14.9 \%$ had a French highschool diploma (12 years of education) and $29.7 \%$ had a graduate diploma (at least 14 years of education).

At S5 (3 years after FCSRT completion) 90 participants had developed dementia (AD $n=57$ ), and at S7 (5 years after FCSRT completion) the cumulative number of incident cases of dementia (over the 3 and 5 years) was 173 (AD $n=116)$.

The coefficients of the linear regressions used to produce the expected FCSRT scores according to age, agesquared, sex and level of education are shown in Additional file 1: Table S1. These models explained from $R^{2}$ $=4.5 \%$ to $R^{2}=10.6 \%$ of the total variance of the different FCSRT scores. The "crude scores", the "residual scores" (differences between crude and estimated FCSRT scores) and the percentage of "refusal or abandon" (subjects who did not begin or complete the neuropsychological test at S4) relative to the occurrence of dementia and $\mathrm{AD}$ at 3 and 5 years are presented in Table 1. Comparisons of the AUC for the ROC curves of the crude and residual scores (Table 2 and Fig. 2a, b) showed that, compared with the crude scores, the residual scores did not improve the prediction of dementia or $\mathrm{AD}$ occurrence at 3 or 5 years, and between 3 and 5 years, whichever FCSRT score was considered.

The construction of the "risk scores" was based on the beta coefficients from logistic regression analyses performed using the Bordeaux center data (training dataset). Each risk score included a given FCSRT score, age, sex, education level and the interaction between these three variables and the FCSRT score. Coefficients of logistic regression used for the construction of risk scores are shown in Additional file 2: Table S2. The performances of the risk scores for the prediction of dementia or $\mathrm{AD}$ were evaluated using the Montpellier center data (testing dataset) (as recommended in [27]). Comparison of the AUC for the risk scores vs crude scores showed that the risk scores did not improve the prediction of dementia and $\mathrm{AD}$ at 3 and 5 years, and between 3 and 5 years (Table 3 ). The sensibility analysis conducted by inverting the cities for the training and testing dataset led to the same conclusion (data not shown).

\section{Discussion}

Here, we show that the use of "risk scores" or "residual scores" that take into account sex, age and education level does not improve the FCSRT performance for the prediction of dementia and AD at 3 and 5 years compared with the crude FCSRT scores. Therefore, the FCSRT on its own, without the addition of sociodemographic data, is sufficient for the prediction of dementia and $\mathrm{AD}$ in the general population.

Our study has several strengths. First, we conducted this analysis on data from the $3 \mathrm{C}$ trial, a large cohort of older subjects recruited from the general population in France. This enabled us to have sufficient numbers of patients who developed dementia or $\mathrm{AD}$ during the follow-up for a prediction study that included a crossvalidation of the risk scores. Second, we used the FCSRT [8]. This neuropsychological test is recommended for assessing amnesic syndrome of hippocampal type [5] and has been frequently reported to be efficient also in the context of AD detection and prediction [9, 30-32]. In agreement, our study confirmed the previous results on FCSRT prediction of dementia in the Bordeaux cohort of the $3 \mathrm{C}[10]$ and showed the better performance of the free recall score for the prediction of dementia and $\mathrm{AD}$, as reported also by the GuidAge study in patients with memory complaints [30]. We used in this study the validated French version of the FCRST [22] which has been used in most French studies analyzing the FCRST (PREAL study [9], Three-City Study [10] or CONSTANCES study [16]). This version differs from the original version in the presentation, during the encoding phase of the test, with cards displaying words $(4 \times 4)$ adapted to the French-speaking population rather than cards displaying pictures. In two French studies $[9,10]$ which have determined a cutoff value to predict dementia, these values were lower than the one proposed by Grobber et al. [33]. More precisely, Sarazin et al. [9] proposed a free recall score $\leq 17$ for the prediction of $\mathrm{AD}$ at 3 years in $\mathrm{MCI}$ subjects, whereas Grobber et al. [33] proposed a score $\leq 24$ to identify very mild dementia 


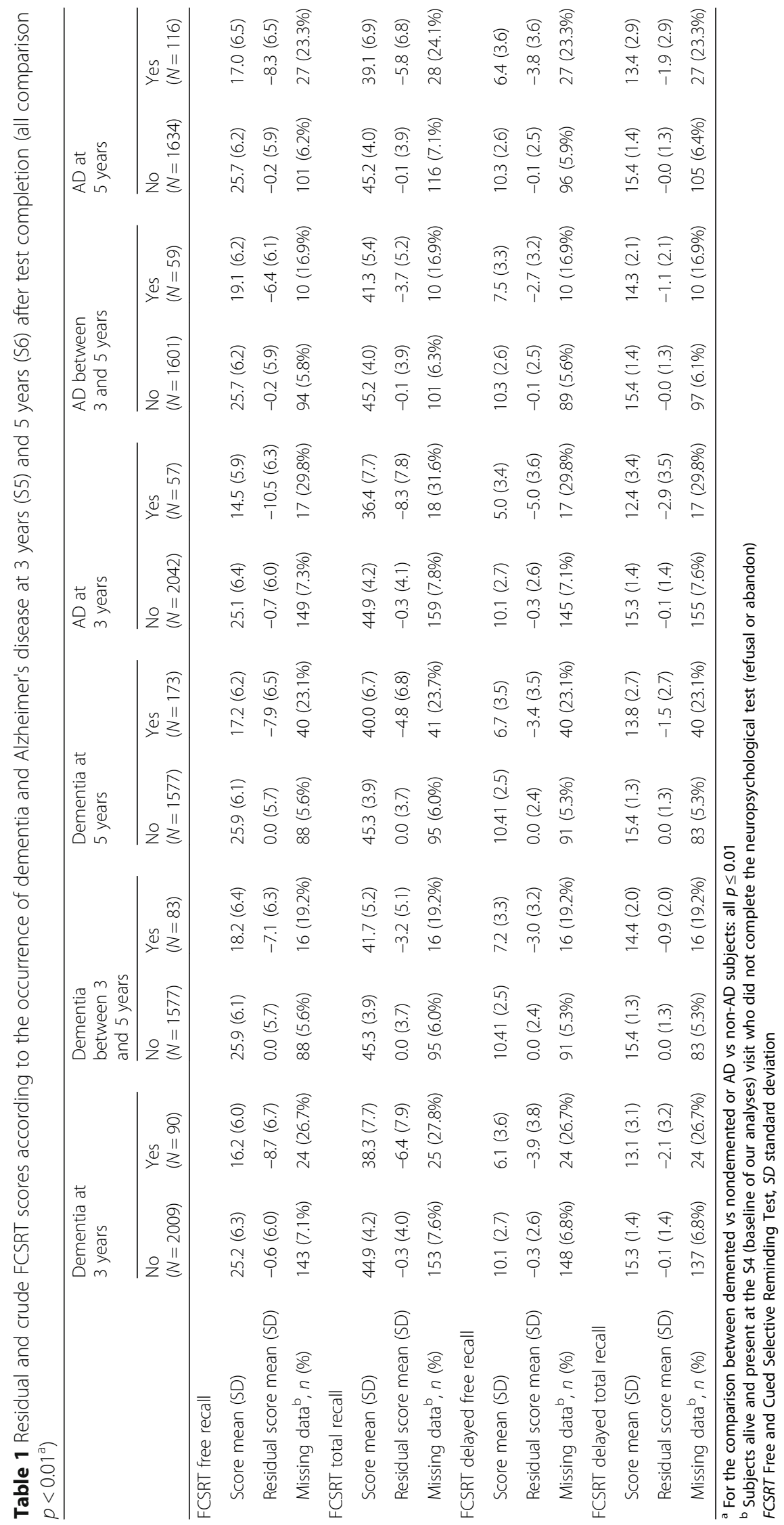




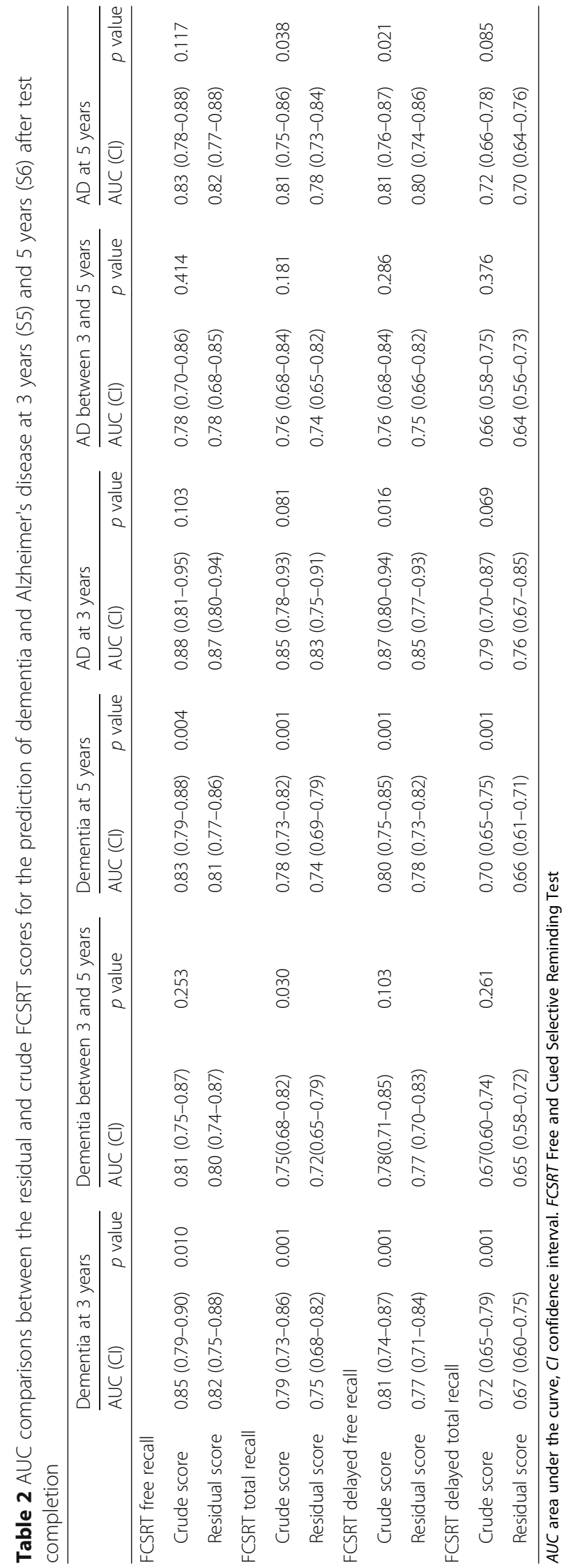


a
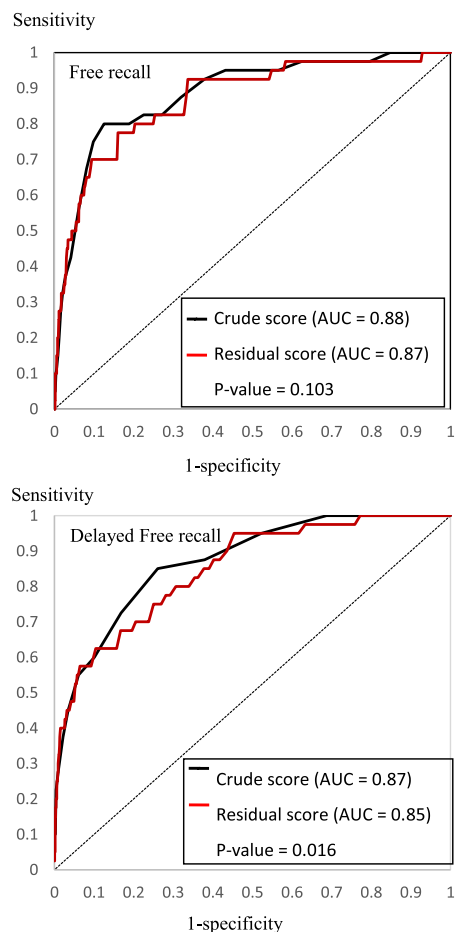

b

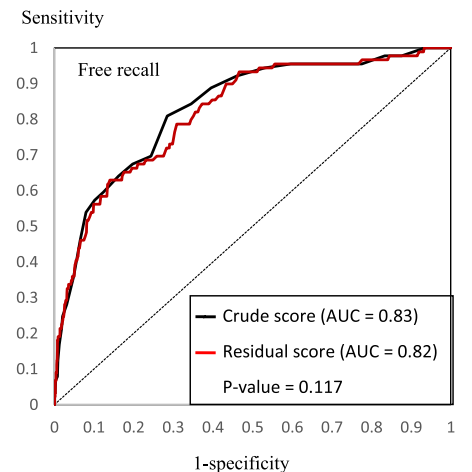

Sensitivity

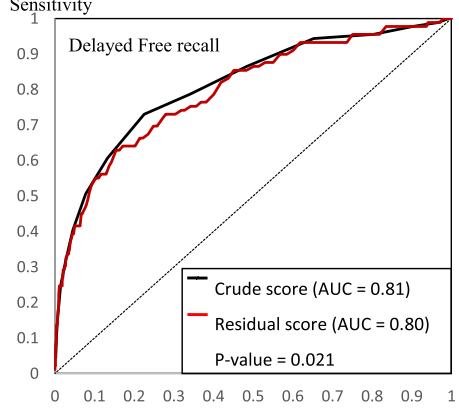

1 -specificity
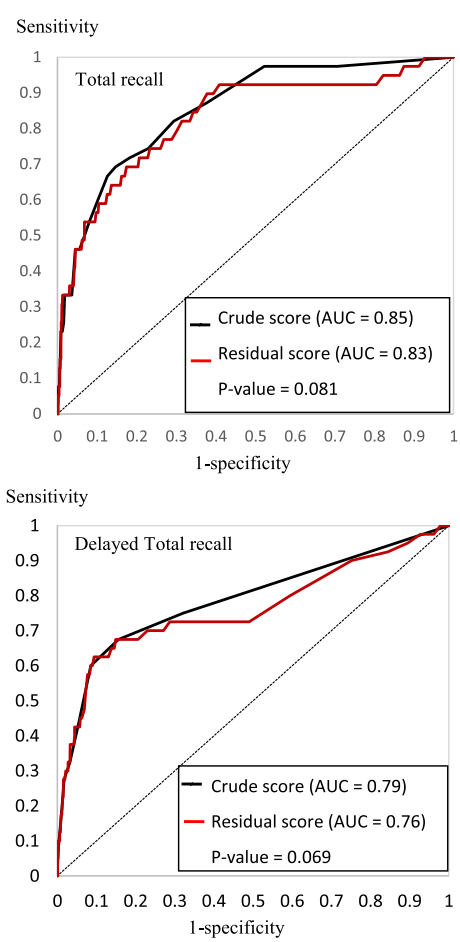

Sensitivity

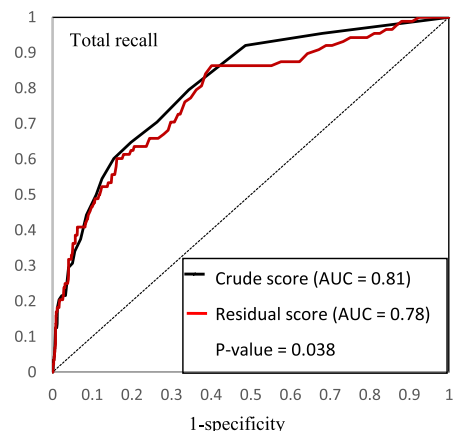

Sensitivity

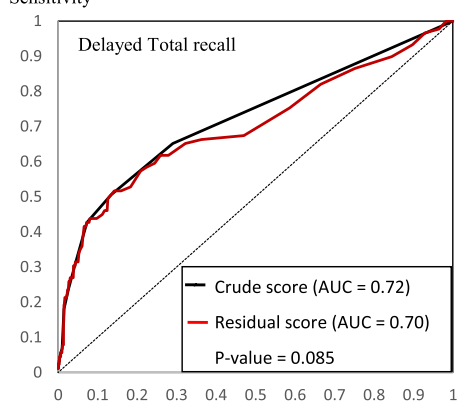

1 -specificity

Fig. 2 ROC curves representing the FCSRT crude and residual scores for the prediction of Alzheimer's disease at a 3 years and $\mathbf{b} 5$ years AUC area under the curve, FCSRT Free and Cued Selective Reminding Test

in primary care. Furthermore, Auriacombe et al. [10] proposed a free recall score $\leq 16$ for the prediction of dementia at 2 years, and a score $\leq 24$ for the prediction at 5 years in the general population. This means that the
French version of the test is calibrated on a more difficult basis than the original version, and explains why subjects who became demented during our study appear to have an initial FCSRT score similar to the scores of 


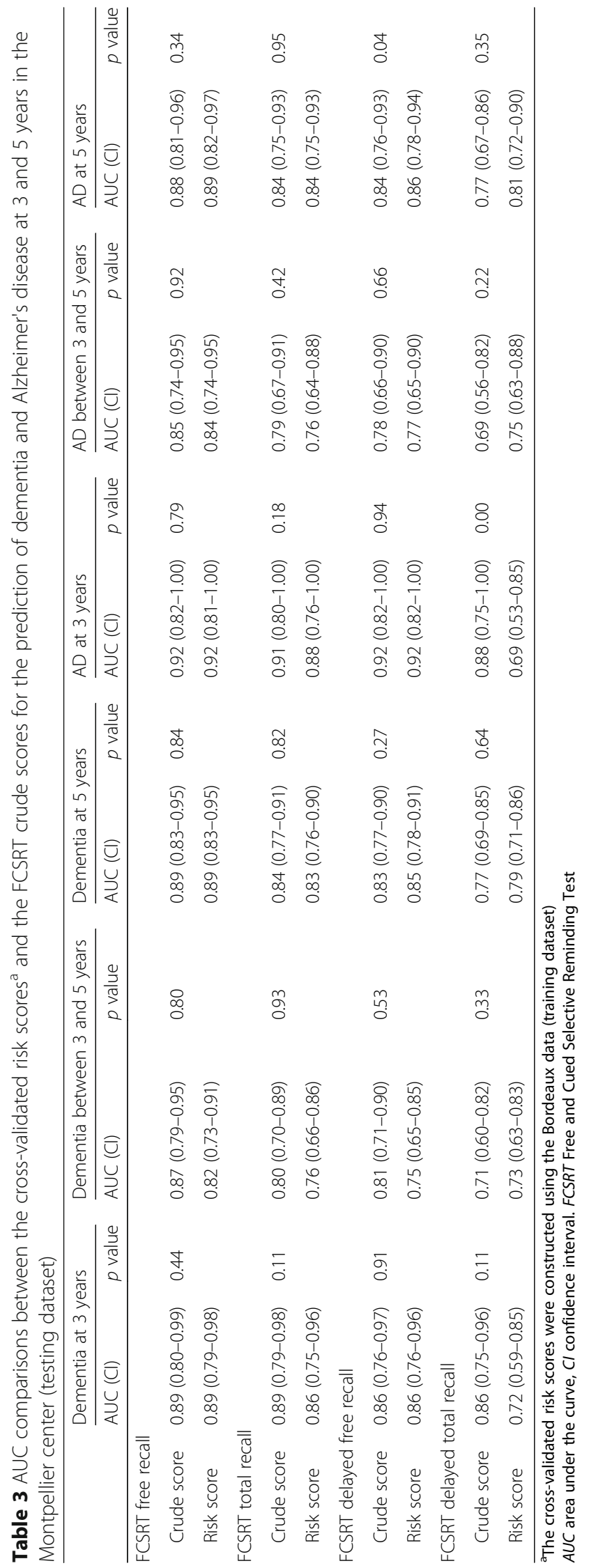


already demented patients in the publication by Grober et al. [33].

A point which could be raised concerning the prediction performances of the "residual scores" is the part of the variance explained by socio-demographics factors. This part varies from $10.6 \%$ for the free recall score to $4.5 \%$ for the delayed total recall score. These percentages are consistent with figures published in the general population [16]. The smaller amount of explained variance for the total recall and delayed total recall scores can be explained by the ceiling effect that affects these two scores. Indeed, if a large number of subjects have the maximum score, the part of variance that might be explained by socio-demographic factors is automatically reduced. Conversely, the free recall and delayed free recall scores do not exhibit a ceiling effect. Another explanation could be the relative homogeneity of our sample (all subjects were older than 70 years) that did not allow fully capturing the cognitive variability between younger and older subjects.

In our study, $7-8 \%$ of participants did not complete the FCSRT test. This should not be considered a limitation of the study. Indeed, the interruption or refusal during the execution of a test is a reality that cannot be avoided in clinical practice. Moreover, our data indicate that almost one-third of noncompleters developed $\mathrm{AD}$ in the next 3 years (Table 1). This behavior could therefore be considered a risk factor for AD. This result is consistent with a previous publication showing that refusing neuropsychological tests is associated with poorer cognitive functioning [34].

For this study, we did not have access to postmortem data to confirm the diagnosis of AD or dementia. Nevertheless, all diagnoses were reviewed by a panel of independent experts who had access to all data, including the FCSRT scores. Consequently, the clinical diagnoses and FCSRT results were not independent, which could have overestimated FCSRT predictive performance. However, it is unlikely that this potential classification bias influenced the comparison of the crude, residual and risk scores. Moreover, for $\mathrm{AD}$ prediction, we grouped subjects with other dementia types in the non-AD group, together with nondemented subjects. This choice might have decreased FCSRT predictive performance, but should not have influenced the comparison between scores.

Concerning the risk scores, their construction and assessment using the same dataset generally leads to overfitting the model and overestimating their performances. Therefore, we split our data into two datasets, based on the inclusion center (Bordeaux and Montpellier), for their construction and cross-validation, as recommended by Altman et al. [27]. This analysis showed that the risk scores did not predict dementia or AD better than the crude scores.
The absence of improvement of the test predictive performances when the socio-demographic variables are taken into account could also bring new insight into the question of the cognitive reserve. Reed et al. [19] suggested including socio-demographic factors in a residual score for $\mathrm{AD}$ prediction because they thought that the neuropsychological test scores should be corrected for age, sex and education to better measure the cognitive reserve. Conversely, our results suggest that this is not the case. Reed et al.'s [19] approach was based on the hypothesis that the cognitive reserve could be better estimated by removing the parts of variability of a neuropsychological test due to socio-demographic features and brain damage load. We did not have information about the severity of brain damage in our population, a major issue in their hypothesis [19]. Nonetheless, our results stress that this hypothesis needs to be further investigated before being accepted.

Another new finding of our study is that FCSRT crude scores exhibit good performance for the prediction of dementia between 3 and 5 years independently of the cases diagnosed in the first 3 years. Although it may seem difficult in practice to differentiate subjects who will convert before and after 3 years, these results explore the maintenance of performance of the neuropsychological test several years after the examination. It will be interesting in future research to study the prediction of dementia and/or $\mathrm{AD}$ even earlier, 5 to 10 years before the diagnosis of dementia. In this context our results will not necessarily apply to this issue and will have to be re-evaluated

A practical implication of our results is that, in the context of a therapeutic trial concerning the early stages of dementia/AD (i.e., before the clinical signs/ diagnosis), the FCSRT could be used alone, without any information about socio-demographic features, to identify the target population at very high risk of dementia and/or AD. It could be used with a single threshold (e.g., a free recall score $\leq 22$, as proposed by Auriacombe et al. [10]), without norms by sex, age and education, despite the strong FCSRT dependence on these features. In the future, other methods, such as the simultaneous use of several cognitive scores or the analysis of FCSRT longitudinal variations during a repeated follow-up, could be explored to improve FCSRT predictive performances.

\section{Conclusions}

Our study shows that the FCSRT predicts dementia and AD independently from socio-demographic characteristics, and that the use of this information does not improve prediction of dementia when using FCSRT results in the general population. 


\section{Additional files}

Additional file 1: is Table S1. presenting coefficients for the linear regression analyzing the relation between FCSRT scores and age, agesquared, sex and education. (DOCX $20 \mathrm{~kb}$ )

Additional file 2: is Table S2. presenting coefficients of logistic regression used for the construction of risk scores of Alzheimer's dementia at 3 years and at 5 years based on age, sex, education, FCSRT scores and the interaction between socio-demographics factors and FCSRT scores. (DOCX 29 kb)

\section{Abbreviations}

AD: Alzheimer's disease; AUC: Area under the curve; FCSRT: Free and Cued Selective Reminding Test; MMSE: Mini Mental State Examination; $R^{2}$ : Coefficient of determination; ROC: Receiver operating curve; SD: Standard deviation

\section{Funding}

The $3 \mathrm{C}$ is conducted under a partnership agreement between the Institut National de la Santé et de la Recherche Médicale (INSERM), the Victor Segalen-Bordeaux II University and Sanofi-Aventis. The Fondation pour la Recherche Médicale funded the preparation and initiation of the study. The $3 C$ is also supported by the Caisse Nationale Maladie des Travailleurs Salariés, Direction Générale de la Santé, MGEN, Institut de la Longévité, Conseils Régionaux of Aquitaine and Bourgogne, Fondation de France and Ministry of Research-INSERM Programme "Cohortes et collections de données biologiques". The funding organizations played no role in the design and conduct of the study; in the collection, management, analysis, and interpretation of the data; or in preparation, review, or approval of the manuscript.

\section{Availability of data and materia}

The datasets used and/or analyzed during the current study are available from the corresponding author on reasonable request.

\section{Authors' contributions}

TM and MB comanaged the literature searches, undertook the statistica analysis, cowrote the first draft of the manuscript and report no disclosures. AG, SA and J-FD comanaged the literature searches, made substantial modifications to the manuscript, approved the final version and report no disclosures. $\mathrm{HA}$ and $\mathrm{CB}$ codesigned the study and wrote the protocol, made substantial modifications in the manuscript, approved the final version and report no disclosures. All authors read and approved the manuscript.

\section{Competing interests}

The authors declare that they have no competing interests.

\section{Consent for publication}

Not applicable.

\section{Ethics approval and consent to participate}

The study protocol was approved by the Ethical Committee of the "KremlinBicêtre" Hospital (France, Paris). All authors of and contributors to the current study have agreed to conditions noted on the Authorship Agreement Form. All subjects enrolled in the study signed an informed consent form after the nature of the procedures was explained fully.

\section{Author details}

1 Neuropsychiatry: Epidemiological and Clinical Research, INSERM U1061, 34093 Montpellier, Cedex 5, France. University of Montpellier, Montpellier, France. ${ }^{3}$ Medical Information Department, University Hospital of Montpellier, Montpellier, France. ${ }^{4}$ Memory Consultation, Centre Mémoire de Ressource et de Recherche, Gui de Chauliac University Hospital, Montpellier, France. ${ }^{5}$ Memory Consultation, Centre Mémoire de Ressource et de Recherche, University Hospital, Bordeaux, France. ${ }^{6}$ ISPED, Centre INSERM U1219, Bordeaux Population Health Research Center, Bordeaux, France. ${ }^{7}$ University of Bordeaux, Centre INSERM U1219, Bordeaux Population Health Research Center, Bordeaux, France.

Received: 5 October 2016 Accepted: 21 December 2016 Published online: 23 March 2017

\section{Reference}

1. Braak H, Thal DR, Ghebremedhin E, Del Tredici K. Stages of the pathologic process in Alzheimer disease: age categories from 1 to 100 years. J Neuropathol Exp Neurol. 2011;70(11):960-9.

2. Amieva H, Le Goff M, Millet X, Orgogozo JM, Peres K, Barberger-Gateau P, Jacamin-Gadda H, Dartigues JF. Prodromal Alzheimer's disease: successive emergence of the clinical symptoms. Ann Neurol. 2008;64(5):492-8.

3. Amieva H, Mokri H, Le Goff M, Meillon C, Jacqmin-Gadda H, Foubert-Samier A, Orgogozo JM, Stern Y, Dartigues JF. Compensatory mechanisms in higher-educated subjects with Alzheimer's disease: a study of 20 years of cognitive decline. Brain. 2014;137(Pt 4):1167-75.

4. Golde TE. Disease modifying therapy for AD? J Neurochem. 2006;99(3):689-707.

5. Dubois B, Feldman HH, Jacova C, Hampel H, Molinuevo JL, Blennow K, DeKosky ST, Gauthier S, Selkoe D, Bateman R, et al. Advancing research diagnostic criteria for Alzheimer's disease: the IWG-2 criteria. Lancet Neurol. 2014;13(6):614-29.

6. Laske C, Sohrabi HR, Frost SM, Lopez-de-Ipina K, Garrard P, Buscema M, Dauwels J, Soekadar SR, Mueller S, Linnemann C, et al. Innovative diagnostic tools for early detection of Alzheimer's disease. Alzheimers Dement. 2015; 11(5):561-78.

7. Pillai JA, Cummings JL. Clinical trials in predementia stages of Alzheimer disease. Med Clin North Am. 2013;97(3):439-57.

8. Grober E, Buschke H, Crystal H, Bang S, Dresner R. Screening for dementia by memory testing. Neurology. 1988;38(6):900-3.

9. Sarazin M, Berr C, De Rotrou J, Fabrigoule C, Pasquier F, Legrain S, Michel B, Puel M, Volteau M, Touchon J, et al. Amnestic syndrome of the medial temporal type identifies prodromal AD: a longitudinal study. Neurology. 2007;69(19):1859-67.

10. Auriacombe S, Helmer C, Amieva H, Berr C, Dubois B, Dartigues JF. Validity of the free and cued selective reminding test in predicting dementia: the 3C study. Neurology. 2010;74(22):1760-7.

11. Singh-Manoux A, Kivimaki M, Glymour MM, Elbaz A, Berr C, Ebmeier KP, Ferrie JE, Dugravot A. Timing of onset of cognitive decline: results from Whitehall II prospective cohort study. BMJ. 2012;344:d7622.

12. Gerstorf D, Herlitz A, Smith J. Stability of sex differences in cognition in advanced old age: the role of education and attrition. J Gerontol B Psychol Sci Soc Sci. 2006;61(4):245-9.

13. Aartsen MJ, Martin M, Zimprich D. Gender differences in level and change in cognitive functioning. Results from the Longitudinal Aging Study Amsterdam. Gerontology. 2004;50(1):35-8.

14. Schneeweis N, Skirbekk V, Winter-Ebmer R. Does education improve cognitive performance four decades after school completion? Demography. 2014;51(2):619-43.

15. Zahodne LB, Glymour MM, Sparks C, Bontempo D, Dixon RA, MacDonald SW, Manly JJ. Education does not slow cognitive decline with aging: 12 year evidence from the victoria longitudinal study. J Int Neuropsychol Soc. 2011;17(6):1039-46

16. Mura T, Amieva H, Goldberg M, Dartigues JF, Ankri J, Zins M, Berr C. Effect size for the main cognitive function determinants in a large cross-sectional study. Eur J Neurol. 2016;23(11):1614-26.

17. Williams JW, Plassman BL, Burke J, Holsinger T, Benjamin S. Preventing Alzheimer's disease and cognitive decline. In: Evidence Report/Technology Assessment. Rockville: AHRQ: Agency for Healthcare Research and Quality; 2010.

18. Moons KG, Royston P, Vergouwe Y, Grobbee DE, Altman DG. Prognosis and prognostic research: what, why, and how? BMJ. 2009:338:b375.

19. Reed BR, Mungas D, Farias ST, Harvey D, Beckett L, Widaman K, Hinton L, DeCarli C. Measuring cognitive reserve based on the decomposition of episodic memory variance. Brain. 2010;133(Pt 8):2196-209.

20. Stern Y. Cognitive reserve in ageing and Alzheimer's disease. Lancet Neurol. 2012;11(11):1006-12.

21. The $3 C$ Study Group. Vascular factors and risk of dementia: design of the Three-City Study and baseline characteristics of the study population. Neuroepidemiology. 2003;22(6):316-25.

22. Van der linden $M$, Coyette F, Poitrenaud J, GREMEM elmd. L'épreuve de rappel libre/rappel indicé à 16 items (RL/RI-16). In: Van der Linden MAS, Agniel A, et les membres du GREMEM, editors. L'évaluation des troubles de la mémoire Présentation de quatre tests de mémoire épisodique (avec leur étalonnage). Marseille: Solal; 2004. p. 25-47.

23. Folstein MF, Folstein SE, McHugh PR. "Mini-mental state". A practical method for grading the cognitive state of patients for the clinician. J Psychiatr Res. 1975;12(3):189-98. 
24. Isaacs B, Kennie AT. The Set test as an aid to the detection of dementia in old people. Br J Psychiatry. 1973;123(575):467-70.

25. American Psychiatric Association. Diagnostic and Statistical Manual of Mental Disorders. 4th ed. Washington, DC: American Psychiatric Association; 1994

26. McKhann G, Drachman D, Folstein M, Katzman R, Price D, Stadlan EM. Clinical diagnosis of Alzheimer's disease: report of the NINCDS-ADRDA Work Group under the auspices of Department of Health and Human Services Task Force on Alzheimer's Disease. Neurology. 1984;34(7):939-44.

27. Altman DG, Vergouwe $Y$, Royston P, Moons KG. Prognosis and prognostic research: validating a prognostic model. BMJ. 2009;338:b605.

28. Hanley JA, McNeil BJ. The meaning and use of the area under a receiver operating characteristic (ROC) curve. Radiology. 1982;143(1):29-36.

29. DeLong ER, DeLong DM, Clarke-Pearson DL. Comparing the areas under two or more correlated receiver operating characteristic curves: a nonparametric approach. Biometrics. 1988:44(3):837-45.

30. Di Stefano F, Epelbaum S, Coley N, Cantet C, Ousset PJ, Hampel H, Bakardjian H, Lista S, Vellas B, Dubois B, et al. Prediction of Alzheimer's disease dementia: data from the guidage prevention trial. J Alzheimers Dis. 2015;48(3):793-804.

31. Lemos R, Maroco J, Simoes MR, Santiago B, Tomas J, Santana I. The free and cued selective reminding test for predicting progression to Alzheimer's disease in patients with mild cognitive impairment: a prospective longitudinal study. J Neuropsychol. 2015. doi:10.1111/jnp.12075. [Epub ahead of print].

32. Mura T, Proust-Lima C, Jacamin-Gadda H, Akbaraly TN, Touchon J, Dubois B, Berr C. Measuring cognitive change in subjects with prodromal Alzheimer's disease. J Neurol Neurosurg Psychiatry. 2014;85(4):363-70.

33. Grober E, Sanders AE, Hall C, Lipton RB. Free and cued selective reminding identifies very mild dementia in primary care. Alzheimer Dis Assoc Disord. 2010;24(3):284-90.

34. Jacamin-Gadda H, Fabrigoule C, Commenges D, Dartigues JF. A 5-year longitudinal study of the Mini-Mental State Examination in normal aging. Am J Epidemiol. 1997;145(6):498-506

\section{Submit your next manuscript to BioMed Central and we will help you at every step:}

- We accept pre-submission inquiries

- Our selector tool helps you to find the most relevant journal

- We provide round the clock customer support

- Convenient online submission

- Thorough peer review

- Inclusion in PubMed and all major indexing services

- Maximum visibility for your research

Submit your manuscript at www.biomedcentral.com/submit 